\title{
Rursus
}

Russus

Poiétique, réception et réécriture des textes antiques

5 | 2010

Les épopées tardives

\section{Écriture épique et édification religieuse dans l'hagiographie poétique (Ve-VIe s.) : les scènes de résurrections}

Sylvie Labarre

\section{OpenEdition}

Journals

Édition électronique

URL : http://journals.openedition.org/rursus/408

DOI : $10.4000 /$ rursus.408

ISSN : 1951-669X

Éditeur

Université Nice-Sophia Antipolis

Référence électronique

Sylvie Labarre, «Écriture épique et édification religieuse dans I'hagiographie poétique (Ve-Vle s.) : les scènes de résurrections », Rursus [En ligne], 5 | 2010, mis en ligne le 11 mars 2010, consulté le 19 avril 2019. URL : http://journals.openedition.org/rursus/408; DOI : 10.4000/rursus.408

Ce document a été généré automatiquement le 19 avril 2019

Rursus 


\title{
Écriture épique et édification religieuse dans l'hagiographie poétique (Ve-VIe s.) : les scènes de résurrections
}

\author{
Sylvie Labarre
}

\section{Introduction}

1 L'hagiographie est le plus souvent un récit en prose, par exemple la Vie d'Antoine par Athanase, la Passion de Perpétue et Félicité, la Vie de saint Martin de Sulpice Sévère. Elle s'écrit en marge de l'histoire, car elle a la fâcheuse réputation de manquer de vérité, tout en ressortissant à la biographie. C'est l'évocation de la vie d'un homme exceptionnel, intermédiaire entre Dieu et les hommes, tantôt plus près de l'un, quand il accomplit des miracles, tantôt plus près des autres, quand il présente des faiblesses. Cependant, elle offre un matériau de choix pour un récit épique : héros hors normes, série de défis, valeurs morales illustrées par de hauts faits, exemplarité... À la fin du IVe s. et au début du Ve s., Paulin de Nole et Prudence ont fait entrer l'hagiographie en poésie, le premier en composant une Laus S. Iohannis et les Natalicia en l'honneur de saint Félix, le second en tressant de ses vers la couronne des martyrs (Peristephanon liber). Mais la première épopée hagiographique latine est due à Paulin de Périgueux au Ve s., suivi au VIe s. par Venance Fortunat. Ils composent l'un et l'autre une Vie de saint Martin en vers, réécriture ou paraphrase de la Vie et du Gallus (= Dialogues) rédigés par Sulpice Sévère du vivant ou peu après la mort du saint, survenue en 397. Ils adoptent un mode épique dont je m'appliquerai à préciser les caractéristiques et les limites. Après avoir examiné selon quels critères l'appartenance de ces œuvres à l'épopée se manifeste, j'étudierai d'abord l'intertextualité épique, puis l'édification religieuse à travers deux récits de résurrection. En effet, aux chapitres 7 et 8 de la Vie de saint Martin, Sulpice Sévère rapporte comment Martin ramène à la vie un catéchumène, puis un jeune esclave. Paulin de Périgueux récrit 
en vers le premier épisode au chant 1, vers 317 à 365, le second, aux vers 366 à 386, Venance Fortunat donne une nouvelle version poétique des deux passages $(1,159-178$; 179-201). Se déploie alors une thématique tout à la fois épique et biblique. À travers le merveilleux, c'est souvent la divinité qui se manifeste dans le récit épique, cependant que le miracle établit la preuve que Dieu agit par l'intermédiaire du saint.

\section{L'appartenance à l'épopée}

Dans quel contexte sont nées ces deux œuvres poétiques? Paulin de Périgueux, dont nous ne savons rien, fut dès l'Antiquité confondu avec Paulin de Nole, son homonyme prestigieux, mort en 431. C'est à la demande de Perpetuus, évêque de Tours, qui donne au culte de Martin son essor entre 458/9 et 488/9, qu'il compose sa Vie de saint Martin. Nous l'apprenons par une lettre-prologue, conservée dans un seul manuscrit, unique témoin également de plusieurs passages, alors que l'œuvre est transmise par une dizaine de manuscrits médiévaux. Il ajoute au récit de Sulpice des miracles posthumes relatés par Perpetuus. Lorsque Venance Fortunat, poète formé en Italie, entreprend autour de 575 de donner une nouvelle version poétique de la Vie de saint Martin, il est l'ami et le protégé de Grégoire, alors évêque de Tours. Il connaît la première transcription poétique donnée par Paulin, et c'est de manière tout à fait consciente qu'il peut rivaliser avec elle.

\subsection{Le cadre général}

Le choix de l'hexamètre dactylique, l'ampleur des œuvres (3622 vers pour Paulin, 2243 pour Fortunat) et la répartition en chants (six pour Paulin, soit la moitié de l'Énéide, quatre pour Fortunat, soit le tiers de l'Énéide) sont les marques les plus évidentes de l'épopée ${ }^{1}$. Ensuite, il s'agit de chanter les hauts faits d'un héros : gesta tam grandia (Paulin, Prologue, 4), sublimia gesta (Paulin, V.M., 1, 313-314), Martini gesta beati (Fortunat, V.M., 1, 45).

Venance Fortunat inscrit lui-même les Vies versifiées de Martin dans la continuité de l'épopée biblique $(1,14-25)$ :

En effet le premier qui, en coupant le texte sacré selon une mesure docile, use des ressources du vers pour chanter l'œuvre de la majesté divine, est Juvencus. Ensuite, la langue du remarquable Sédulius brilla aussi et Orens effleura quelques sujets de sa bouche fleurie. Le clairvoyant Prudence dédie avec clairvoyance, leur adressant ces offrandes sacrées, aux pieux martyrs, le récit de leurs actes. Paulin, qui se distingue par la noblesse de sa naissance, par son cœur, par sa foi et par son art, développa en vers l'enseignement du maitre Martin. Puis la belle éloquence du poète Arator sillonna ce qu'on nomme les faits et les actes des Apôtres. Quant à l'évêque Alcimus Avitus, il raconta, avec une admirable finesse, la descendance sacrée que jadis rédigea l'auteur de la Genèse.

5 Il cite deux auteurs d'une épopée biblique inspirée par le Nouveau Testament, Juvencus et Sédulius, un auteur d'épopée fondée sur la Genèse et l'Exode, Avit, et Arator qui transposa les Actes des Apôtres.

6 Paulin et Fortunat imitent les épopées antérieures selon une pratique qui a toujours eu cours dans l'Antiquité :

Les Anciens, eux, n'étaient pas dupes: ils disaient qu'un auteur écrivant une nouvelle épopée engageait un combat avec ceux qui le précédaient, il imitait les épopées précédentes, rivalisait sur chaque lieu, empruntait à des genres extérieurs 
comme la tragédie et l'élégie, tissait son texte ; tout discours nouveau s'élaborait à partir des discours anciens. Le travail de l'écriture apparaissait comme un travail de transformation des textes préexistants².

7 En effet l'écriture épique repose sur la réutilisation de matériaux communs. L'imitation de Virgile est tout à fait essentielle, car l'Énéide fournit tout à la fois des expressions formulaires, un merveilleux d'une extraordinaire puissance évocatrice, les sentiments que peut et doit éprouver un héros, comme je l'ai montré dans le chapitre 6 du Manteau partagé (1998). Des transitions temporelles évoquant l'alternance du jour et de la nuit donnent une emphase épique au récit. Paulin de Périgueux se souvient du flambeau de l'aurore (1, 168-169), Fortunat n'a pas oublié le char de la nuit, parvenu au milieu de sa course $(2,244-246)$. Les comparaisons homériques ou les adjectifs composés ${ }^{3}$ donnent au récit hagiographique une résonance épique immédiatement reconnue par le lecteur.

\subsection{La demande d'inspiration}

8 Virgile est donc pour les poètes chrétiens un modèle à admirer et à imiter, mais pour obtenir l'inspiration, Paulin en appelle à Martin et au Christ. Avant de relater le miracle de la résurrection du catéchumène, il consacre 15 vers à une demande d'inspiration (1, 298-312) :

À présent, pour peu que nous reprenions les vers des anciens poètes, au moment où soudain des prodiges secoueraient leurs sens étonnés, (300) leur folie insensée invoquerait le misérable Phébus et ne laisserait même pas en repos les Muses trompeuses; sous l'effet des mensonges d'Apollon, son temple de Delphes serait secoué, toute la foule des sourdes Sœurs se laisserait emporter, et ces monstres, une fois convoqués, par leur violence porteraient à son comble toute cette fureur. (305) Mais nous qui demandons au Christ nos idées et nos mots, ô toi qui adores le Christ, puisses-tu nous inspirer quelque peu, Martin, nous t'en prions. Toi qui aux morts as pu rendre la vie, soutenu par l'aide du Seigneur, rends-moi le salut: moi, je serai le premier à réaliser le miracle d'une vie rachetée, (310) le premier à briser les barrières du sépulcre fétide. C'est une juste prière que j'adresse, déjà mort en mon cœur tout entier, au saint patron: que mon âme, revenue à la vie, dise les titres d'une si grande gloire.

9 Il oppose les poètes païens et les poètes chrétiens. Les anciens poètes (ueteres uates) invoquaient le misérable Phébus (miser Phoebus), de fausses Muses (falsae Musae), Apollon qui n'est qu'un menteur (mentitus Apollo) et les sourdes Soeurs (surdae Sorores), dès qu'ils avaient un prodige à raconter. Or lui, il s'adresse à Martin lui-même: Christicola, inspires paulum, Martine, precamur. Cependant il n'est pas vrai qu'il appelle Martin mea Musa en 4, 246. Il ne s'agit là que d'une conjecture de Th. Wopkens, tandis que les manuscrits portent la leçon uia uersa, «j'ai changé de route », car rejeter les muses antiques c'est emprunter une voie nouvelle.

10 Cette demande d'inspiration ménage une pause à l'intérieur du récit, donne une certaine solennité au passage qui va suivre et indique clairement la rivalité qui s'engage avec les épopées païennes. Quant à Fortunat, il fait de son long poème une offrande due au saint $(1,42-44)$ :

Il convient en effet - une raison majeure l'a exigé - que je m'acquitte de ma dette en chantant le panégyrique du pontife qui fut à l'origine de ma venue en ce pays.

11 Les deux Vies de saint Martin en hexamètres dactyliques se rattachent incontestablement au genre épique. Pour plus d'exactitude, il convient de reconnaitre qu'elles constituent un sous-genre ou une sous-catégorie 4 à l'intérieur de l'épopée, car elles mêlent à elle des 
éléments du panégyrique, introduisent des prières ou ressemblent parfois à un sermon. Cependant l'épopée antique, virgilienne ou ovidienne, n'est déjà pas sans mélanges.

Un débat récent a opposé deux spécialistes italiens de l'Antiquité tardive, Franca Ela Consolino, dans un article intitulé « Il senso del passato : generi letterari e rapporti con la tradizione nella 'parafrasi biblica' latina $»^{5}$, et Antonio V. Nazzaro', dans une réfutation intitulée "Riscritture metriche di testi biblici e agiografici in cerca del genere negato ", pour savoir si les épopées bibliques et hagiographiques constituaient un genre à part entière, la première affirmant que non, le second soutenant que oui, en repoussant les uns après les autres les arguments de sa collègue. A. V. Nazzaro est tout à fait convaincant, même si la démonstration de Fr. E. Consolino est riche et solide.

\subsection{La transposition de l'aventure épique}

13 L'épopée romaine connut dans l'Antiquité une incontestable mutation, depuis Livius Andronicus et Naevius qui écrivaient en vers saturniens jusqu'à l'épopée flavienne. Elle vit s'opérer un déplacement depuis les thèmes épiques grecs attachés à la guerre de Troie jusqu'à l'histoire romaine contemporaine, que ce soit la guerre punique pour Naevius ou la guerre civile pour Lucain. Avec l'épopée biblique, l'aventure épique prend une définition différente selon les deux Testaments. Quant au schéma hérö̈que de l'hagiographie, il est plus proche de celui de l'Énéide que des épopées flaviennes. Martin s'avance comme un héros solitaire. On observe une gradation dans les épreuves, du matériel au spirituel, tout au long de la vie du saint. Martin, comme Énée, ne subit pas d'évolution. Autour d'eux, le monde change, les hommes changent, mais ils demeurent fidèles à leur mission et renferment en eux, à tout moment de leur vie, les qualités qui sont les leurs?

La scène de miracle correspond justement à une mise à l'épreuve du héros. Les deux récits de résurrection obéissent au même schéma : cris d'une foule autour d'un corps inanimé, Martin s'avance, il renvoie tout le monde, ranime le défunt, puis l'efficacité du miracle convainc toute l'assistance de l'efficacité de la puissance divine. Le récit de faits transcendants est lui-même une marque de la poésie épique.

\section{L'intertextualité épique}

Les réminiscences de Virgile, mais aussi de Lucrèce et de Lucain, introduisent un dialogue entre le contexte de départ (hypotexte) et le contexte d'arrivée (hypertexte) et mettent en évidence la prédominance du modèle épique antique.

\subsection{Les ravages de la maladie}

Dans la première scène de résurrection, Martin sauve un catéchumène, c'est-à-dire un candidat au baptême. En deux vers, il décrit les ravages de la maladie qui l'a emporté (1, v. 320-321). Pour cela, il puise au fonds de la poésie hexamétrique latine. Febris totas penitus depasta medullas rappelle la fièvre qui s'attaque aux troupeaux touchés par la gale dans les Géorgiques : artus depascitur ... febris (III, 458). Mais le feu qui dévore les moelles est aussi celui de la passion amoureuse, comme l'amour inspiré par Vénus à Vulcain8. Troubles de l'âme et troubles du corps s'y confondent. Extorquere uitam (v. 321) vient de la description de la peste à Athènes, dans l'épopée didactique de Lucrèce, et traduit la 
brutalité et la violence de la mort ${ }^{9}$. Paulin emploie d'ailleurs de nouveau cette expression dans le second épisode de résurrection ${ }^{10}$.

L'imitation épique des scènes de résurrection est constamment visible de par les nombreuses citations de Virgile : on en compte six dans l'épisode de la résurrection du catéchumène (Paulin, VM, 1, 317-365), cinq dans celle du petit esclave (1, 366-386), une encore, dans celle d'un enfant près de Chartres $(4,254-291)$. L'émotion suscitée par la mort du catéchumène prend une grandeur antique à travers ce jeu de l'intertextualité. Les cris funèbres de la communauté des frères, réunis autour du catéchumène, constituent une forme de planctus épique qui connaitra un grand succès au Moyen Âge, en se développant à l'intérieur du roman. Le génitif pluriel maerentium fratrum dont Sulpice se contentait est développé par Paulin : «mais la piété empressée de ses frères pleurait avec affliction sur le cadavre inanimé, s'affligeant du supplice d'un frère, non de la mort de sa chair ${ }^{11}$ ». Le choix de l'abstrait " piété empressée » (sedula religio), sujet du verbe "pleurer ", un trait de la langue poétique tardive, combine goût pour l'abstraction et représentation sensible. La réécriture poétique fait entendre les cris du planctus, grâce à la figure de l'adnominatio (maesta maerens) qui joue sur la répétition des mêmes sonorités. Les larmes de Martin sont celles d'Énée, lorsqu'il rencontre son père aux Enfers ${ }^{12}$, et ses gémissements ceux d'Andromaque, quand elle reconnaît Énée près de Buthrote ${ }^{13}$. Le corps privé de vie (exanimum corpus) rappelle aussi bien Lucrèce $(6,705)$ que Virgile (Énéide, 6, 149).

Dans le récit de la seconde résurrection, Paulin décrit le cadavre de l'esclave de Lupicinus, en empruntant à l'Énéide l'évocation des stigmates de la mort : « les yeux exorbités et la gorge tarie de sang ${ }^{14} »$. Cette citation n'est pas un pur écho verbal, mais elle suppose un rapprochement des deux contextes. Au livre 8 de l'Énéide, Hercule étreint Cacus et lui fait jaillir les yeux hors de la tête, tandis que sa gorge se vide de son sang. Martin, quant à lui, serre dans ses bras le petit esclave aux yeux exorbités et à la gorge desséchée pour faire fuir la mort. Le saint participe donc à une lutte épique contre des forces maléfiques, comme Hercule débarrasse les populations des monstres qui les terrifient. Prudence lui aussi s'inspire de ces vers, lorsqu'il dépeint le combat de la Foi contre l'Idolâtrie ${ }^{15}$. L'allégorie chrétienne et l'hagiographie puisent leur expression aux mêmes sources de l'épopée antique.

19 En 1, 379, Paulin décrit d'autres manifestations du trépas. Le « regard extrêmement farouche » (lumina torua nimis) rappelle celui des cyclopes (Énéide, 3, 677 : lumine toruo). Il a suffi de changer un singulier en un pluriel. Le "visage menaçant dans la mort " ( uultumque in morte minacem)reprend la fixité effrayante des yeux des cadavres chez Lucain. Le passage où les mères étreignent les cadavres de leurs enfants a marqué l'esprit des lecteurs de la Guerre civile (2, 20 sv.), car il représente une scène de planctus exemplaire. Ces expressions épiques ont une grande puissance évocatrice.

\subsection{Les moyens de la lutte}

20 Comment un poète du Ve s. se représente-t-il un miracle chrétien, et plus précisément un saint qui redonne la vie à un défunt, en s'étendant sur son corps inanimé ? C'est l'épopée antique qui lui fournit les représentations adéquates. Dans l'hypotexte en prose de Sulpice Sévère, Martin était visité par l'Esprit, comme la pythie Phémonoé possédée par Apollon, chez Lucain ${ }^{16}$. Martin s'associe lui-même au planctus, en répandant des larmes abondantes et en poussant des cris (Sulpice, 7, 2 ; Paulin, 1, 325-327 ; Fortunat, 1, 164). 
21 Le procédé qui permet la résurrection rappelle aussi un passage épique. Chez Paulin, quand il est étendu sur le catéchumène, le saint fait inévitablement penser à l'épisode au cours duquel Nisus tombe mort sur le corps de son ami Euryale, car c'est un vers entier de l'Énéide $(9,444)$ qui est reproduit: Tum super exanimum sese proiecit amicum $(1,329)$. Le dialogue des deux contextes ajoute un lien affectif entre Martin et le catéchumène, qui n'est présent ni chez Sulpice, ni chez Fortunat. Le topos de l'amitié qui va jusqu'au sacrifice de soi se trouve d'ailleurs dans un autre passage de la Vita Martini de Paulin, lorsque Martin reprend les traces d'Hilaire ${ }^{17}$. Cependant, le saint ne tombe pas mort sur le corps de son ami, et plutôt que de mourir à ses côtés, il le ressuscite. Il lutte avec les armes de la spiritualité (1, 332-334) :

C'est vers le Seigneur que son espoir tout entier se reporte : l'habituelle pitié est sollicitée, la miséricorde bien connue est réclamée, et c'est en croyant que Martin mérite ce que lui mérite sa foi.

\subsection{La victoire sur la mort}

Chez Paulin, le cliché virgilien nec mora $(1,337)$ traduit la rapidité du résultat obtenu. Puis la résurrection apparaît comme une lutte entre des éléments contraires, l'inertie et le mouvement (329, exanimum cadaver / 338, incussis membris), le dessèchement et la circulation du sang qui revient (339, arentes artus uenarum umore rigantur), le froid et la chaleur (330, frigentes...artus / 340 , pectus glaciale tepescit) , ce qui est clos et ce qui s'ouvre (341, obducti...reserantur uisus).

Quant à Fortunat, il représente la mort comme le Tartare. Martin exige de lui qu'il recrache, tel un monstre mythologique, le défunt : reuomant ut Tartara functum $(1,168)$. Homère et Hésiode désignaient ainsi la partie la plus profonde du monde, située sous l'Hadès (Iliade, 8, 13; Théogonie, 720). Le Tartare s'est ensuite confondu avec les Enfers dans l'épopée latine. La forme neutre pluriel Tartara se trouve chez Lucrèce $(3,42)$, Virgile, (Énéide, 4, 243). Valerius Flaccus appelle Pluton Tartarus pater $(4,258)$. Les poètes chrétiens, à leur tour, sont nombreux à avoir assimilé la mort et le Tartare. Arator emploie souvent de telles expressions ${ }^{18}$. Cependant cet emploi est dénué de signification théologique ${ }^{19}$.

24 Fortunat n'introduit qu'une très légère variation entre les deux épisodes de résurrection : 1, 168: reuomant ut Tartara functum et 1, 188: euomuitque uorax auida de fauce rapinam. Cet emploi de euomere ou reuomere rapproche les résurrections opérées par Martin de la résurrection de Lazare chez Prudence ${ }^{20}$. La mort est traitée par Fortunat comme une allégorie. Devant Martin, elle rebrousse chemin à la manière des démons : "la mort rebroussa chemin, retourna sur ses pas, s'enfuit en abandonnant sa proie et vomit la capture qu'elle dévorait hors de sa gueule vorace ${ }^{21} »$.

Tous ces réemplois ont une valeur allusive : «le poète fait appel à la culture du lecteur et suggère un contexte par une expression caractéristique, évoque un thème ou rappelle un exemple ${ }^{22}$ ». Ils aident le lecteur à mieux se représenter la scène pour en méditer tout le sens spirituel.

\section{Un enseignement théologique}

Car les Anciens cherchaient dans l'épopée une leçon de vie, comme le montre Horace ( Épitres, 1, 2, 2) : 
J'ai relu à Préneste l'écrivain de la guerre de Troie : ce qui est beau, ce qui est laid, ce qui est profitable, ce qui ne l'est point, il nous le dit plus pleinement et mieux que Chrysippe et que Crantor. ont redonné la vie à des enfants ${ }^{27}$. Il s'inscrit donc à la suite des grandes figures de l'Ancien Testament. En effet, Élie s'étend sur le corps du fils de la veuve de Sarepta ${ }^{28}$, comme Élisée sur l'enfant de la Sunamite ${ }^{29}$. Cette manière de faire est intéressante à cause des commentaires exégétiques qu'elle a provoqués de la part d'Ambroise et Jérôme ${ }^{30}$. Ils interprètent tous les deux ce passage dans le même sens. Élisée, en s'étendant, meurt d'une certaine façon, pour ressusciter l'enfant, comme le Christ meurt pour que les hommes reçoivent la vie éternelle. Ainsi la résurrection opérée par Martin s'inscrit dans tout un contexte scripturaire qui lui donne relief et signification. Martin meurt d'un point de vue symbolique pour redonner vie au catéchumène et se place dans la lignée des prophètes ou dans celle du Christ. 

paraphrastique ajoute un commentaire moral et spirituel. Ce qui provoque la douleur de la communauté n'est pas l'anéantissement de la chair, mais le supplice d'un frère $(1,324)$. La résurrection est aussi une renaissance spirituelle, l'aube d'un jour nouveau. Cette idée est sensible dans les indications de lumière ${ }^{36}$. Par une tournure proverbiale et virgilienne (felices quibus...contigit...), Paulin donne une portée générale à l'expérience qui est offerte au catéchumène. Tout autre à qui il serait donné de voir Martin éprouverait pareil bonheur. De même, le souffle qui régénère le défunt s'inscrit dans la continuité d'une création divine qui se poursuit au-delà de l'oeuvre des six jours, c'est le souffle de Dieu qui a créé Adam ${ }^{37}$. Afflatus se trouve en ce sens chez Tertullien et a pour synonyme spiraculum dans la Genèse ${ }^{38}$. interprétation du récit. Son but n'est pas simplement l'amplification poétique. Il distingue le cœur pur (puri fiducia cordis), qui permet de voir Dieu, et la chair (uiscera) : "La confiance de son cœur pur a vu que Dieu était là, et sa chair en a senti l'effet dans ses pieuses entrailles » $(1,335-336)$. Seuls les « cœurs purs » verront Dieu, car ils possèdent les « yeux du cœur » dont parle saint Paul : «Puisse-t-il illuminer les yeux de votre cœur pour vous faire voir quelle espérance vous ouvre son appel, quels trésors de gloire renferme son héritage parmi les saints " (Éph. 1, 18) $)^{39}$. On reconnaît chez Paulin, à l'état diffus, la doctrine des sens spirituels développée par Origène : «Et ce qui voit Dieu, c'est le cœur pur ${ }^{40} »$.

Le catéchumène revient à la vie progressivement. Ce retour est exprimé en un vers, fait de quatre membres sensiblement égaux ${ }^{41}$, composés d'un nom et d'un adjectif. Les poètes tardifs affectionnent le tetracolon. Paulin juxtapose les différents signes de la vie: l'activité de la voix, du pas, de la main et du cœur. Mais là, il ajoute un commentaire et passe ainsi de l'idée du corps qui revit dans toute sa réalité physiologique, à une réflexion plus abstraite : «l'homme tout entier, avec son corps formé de l'assemblage de différents principes, reçut une vie une en ses fonctions diverses ${ }^{42}$ ». Vnam uitam renvoie à l'unité spirituelle, alors que la réalité corporelle est dispersion. Cette idée néoplatonicienne est développée par Augustin dans les Confessions : « et ces diverses fonctions (entendre, voir), 
par eux (les sens) je les accomplis en restant un, moi l'esprit ${ }^{43} »$. C'est le dépassement de la vie organique et sensible qui permet de connaitre Dieu. Toutefois la référence la plus importante reste saint Paul, qui compare l'Église et ses membres au corps : «De même, en effet, que le corps est un, tout en ayant plusieurs membres, et que tous les membres du corps, en dépit de leur pluralité, ne forment qu'un seul corps, ainsi en est-il du Christ » (1 Cor. 12, 12); ou encore : « Aussi bien le corps n'est-il pas un seul membre, mais plusieurs » (1 Cor. 12, 14).

\section{3 Écriture épique et écriture épigrammatique}

Plutôt que d'introduire un commentaire moral et spirituel, Fortunat, quant à lui, métaphorise les événements. On pourrait dire que sa translatio, au sens de transcription en vers, est une metaphora.

Il exclut toute recherche excessive dans le vocabulaire pour créer des jeux formels ${ }^{44}$. Il résume ainsi l'action de Martin : et premit arca sacri hunc ne premat arca sepulchri $(1,185)$. Il joue sur la répétition du verbe premere à deux modes différents, d'abord l'indicatif, puis le subjonctif. Une rime interne (sacri / sepulchri) partage le vers en deux hémistiches. On pourrait traduire : «l'arche du saint l'étreint, pour que l'arche du tombeau ne l'étreigne pas ». En s'étendant sur le jeune esclave, Martin l'étreint. Mais c'est aussi l'arche de son cœur, son affection, qui l'enserre. Le poète joue sur les différents sens ou emplois de arca. Le sens premier de ce mot est d'abord le coffre, qui peut-être cercueil ou tombeau. Le premier arca signifie la protection spirituelle procurée par le saint, tandis que le second arca désigne proprement le tombeau réél. Le poète formule un paradoxe : l'arche sauve de l'arche. Dans l'épitaphe de Gallus de Clermont, il joue de la paronomase, procédé qui fait entendre non pas la répétition du même mot, mais deux mots aux sonorités très proches, urna (l'urne) et ulna (le bras) : non premit urna rogi, sed tenet ulna Dei, «ce n'est pas l'urne funéraire qui l'étreint, mais le bras de Dieu qui le tient » (Poèmes, 4, 4, 32). La paronomase me semble impossible à traduire. Ce rapprochement montre la virtuosité de Fortunat dans la forme brève. Or, dans son épopée martinienne, il atteint souvent cette réussite poétique.

Il emprunte quelques mots-clés de la résurrection à des auteurs chrétiens. Rediuiuus (1, 177) se trouve chez Cyprien, à propos de la résurrection de Tabitha par Pierre, chez Prudence, appliqué au Christ, et chez Sédulius, dans la résurrection de Lazare ${ }^{45}$. Cet adjectif signifie à la fois la résurrection du corps et la naissance à la vie spirituelle. Fabrica , qui signifie la charpente, est employé par les chrétiens pour désigner l'homme ou le corps humain tout entier ${ }^{46}$. Le poète recherche une formulation dense, capable de surprendre son lecteur. L'énoncé paradoxal est un bon moyen d'y parvenir. Ainsi l'esclave de Lupicin fait-il des efforts pour reconduire jusqu'au vestibule de la maison celui qui l'a soudain arraché, déjà défunt, au seuil de la mort ${ }^{47}$. La résurrection du catéchumène se résume en un paradoxe plus compliqué : il se survit à lui-même et il est «à la fois l'auteur de ses jours et son propre héritier ${ }^{48} »$. Ainsi la mère de Martin, parce que son fils la convertissait au christianisme, était la mère et la fille de son propre enfant, qui lui donnait une nouvelle naissance ${ }^{49}$. Ce paradoxe figurait déjà chez Paulin de Périgueux ${ }^{50}$. Des expressions semblables se rencontrent fréquemment à partir du concile d'Éphèse, à propos de Marie, qui est mère et fille du Christ, car celui-ci est co-éternel au Père créateur ${ }^{51}$. 
39 L'écriture épique de Fortunat est fortement influencée par l'épigramme, qu'il pratique par ailleurs avec virtuosité.

\section{Conclusion}

40 L'épopée hagiographique est une épopée à laquelle se mêlent des éléments du panégyrique ou du sermon. C'est une poésie qui a des ambitions littéraires, qui cherche à charmer (delectare), émouvoir (mouere) et instruire (docere), tout en tendant vers une finalité, comme toute épopée d'ailleurs. L'utilité fonctionnelle de l'hagiographie versifiée s'inscrit parfaitement dans la tradition romaine. L'objectif de Virgile était de défendre et propager une foi dans la mission providentielle des Romains. Paulin de Périgueux et Venance Fortunat, quant à eux, assurent la promotion du culte de Martin. Leurs épopées sont un acte de louange adressé à Dieu, une prière d'action de grâces, une demande d'intercession.

BIBLIOGRAPHIE

\section{Éditions}

PAULIN DE PÉRIGUEUX, CEuvres, éd. et trad. de E.-F. Corpet, Paris, 1849, p. 5-230.

PAUlin de PÉRIGUeuX, Carmina, éd. M. Petschenig, CSEL 16, pars 1, Wien, 1888.

SULPICE SÉVÈRE, Vie de saint Martin, intro., éd., trad. et commentaire de J. Fontaine, SC 133-135,

Paris, 1967-1969.

SULPICE SÉVÈRE, Gallus. Dialogues sur les « vertus » de saint Martin, éd., trad. et notes de J. Fontaine, SC 510, Paris, 2006.

VenANCE FoRTUNAT, Vie de saint Martin, éd. et trad. de S. Quesnel, CUF, Paris, 1996.

\section{Études critiques}

BUREAU Bruno, Lettre et sens mystique dans l'Historia apostolica d'Arator, Exégèse et épopée, Paris, 1997, p. 20-31.

CHARLET Jean-Louis, « Aesthetic Trends of Late Latin Poetry (325-410) », Philologus, 132 (1988), 1, p. 74-85.

CHARLET Jean-Louis, L'épopée latine aux IVème et Vème siècles, in Aspetti della poesia epica latina, Atti del corso di aggiornamento per docenti di latino e greco del Canton Ticino, Lugano, 1993, a cura di C. Reggi, Lugano, 1995, p. 207-225.

CHARLET Jean-Louis, « Tendances esthétiques de la poésie latine tardive (325-470) », Antiquité

tardive, 16 (2008), p. 159-167. 
CONSOLINo Franca Ela, « Il senso del passato : generi letterari e rapporti con la tradizione nella 'parafrasi biblica' latina ", in Nuovo e antico nella cultura greco-latina di IV-VI secolo, a cura di Isabella Gualandri, Fabrizio Conca, Raffaele Passarella, Quaderni di Acme 73 (2005), Milano, p. 447-526.

CURTIUS Ernst Robert, La littérature européenne et le Moyen Âge latin, trad. française par Jean Bréjoux, Paris, 1956 (éd. allemande, 1947, rééd. 1986, 2 tomes).

CUTINo Michele, L'Alethia di Claudio Mario Vittorio. La parafrasi biblica come forme di espressione teologica, Roma, 2009.

DEPROosT Paul-Augustin, L'apôtre Pierre dans une épopée du VI ${ }^{e}$ siècle : l'Historia apostolica d'Arator, Paris, 1990.

DEPROOST Paul-Augustin, «L'épopée biblique en langue latine. Essai de définition d'un genre littéraire », Latomus, 56, 1, janvier-mars 1997, p. 14-39.

DEPROOST Paul-Augustin, « Ficta et facta. La condamnation du « mensonge des poètes » dans la poésie latine chrétienne », REAug 44 (1998), p. 101-121.

FONTAINE Jacques, Naissance de la poésie dans l'Occident chrétien, Paris, 1981.

GREEN Roger P. H., Latin Epics of the New Testament, Juvencus, Sedulius, Arator, Oxford, 2006.

HERZOG Reinhart, Die Bibelepik der lateinischen Spätantike, Formgeschichte einer erbaulichen Gattung, t. 1, München, 1975.

KARTSCHOKE Dieter, Bibeldichtung : Studien zur Geschichte der epischen Bibelparaphrase von Juvencus bis Otfrid von Weissenburg, München, 1975.

KIRSCH Wolfgang, Die lateinische Versepik des 4. Jahrhunderts, Berlin, 1989.

KIRSCH Wolfgang, Laudes Sanctorum. Geschichte der hagiographischen Versepik vom IV. bis X.

Jahrhundert, I/2, Stuttgart, 2004, p. 312-365.

LABARRE Sylvie, Le manteau partagé: deux métamorphoses poétiques de la Vie de saint Martin chez Paulin de Périgueux ( $V^{e}$ siècle) et Venance Fortunat (VI siècle), Collection des Études Augustiniennes, série Antiquité, 158, Paris, 1998.

LABARRE Sylvie, « La méditation spirituelle dans la poésie hagiographique latine : le cas de Paulin de Périgueux (Ve s.) ", in Motivi e forme della poesia cristiana antica tra scrittura e tradizione classica, XXXVI Incontro di studiosi dell'antichità cristiana, (Roma, 3-5 maggio 2007), Studia Ephemeridis Augustinianum, 108, Roma, 2008, p. 579-588.

LABARRE Sylvie, «Le projet poétique des auteurs latins d'épopées bibliques : la place des ekphraseis ", in Manifestes littéraires dans la latinité tardive (colloque international organisé par P. GalandHallyn et Vincent Zarini à l'Université Paris - Sorbonne, 23-24 mars 2007), Collection des Études Augustiniennes, série Antiquité, 188, 2009, p. 35-50.

MADÉLÉNAT Daniel, L'épopée, Paris, 1986.

NAzZARo Antonio V., «L'agiografia martiniana di Sulpicio Severo e le parafrasi epiche di Paolino di Périgueux e Venanzio Fortunato ", in Mutatio rerum, Letteratura Filosofia Scienza tra tardo antico e altomedioevo, Atti del Convegno di Studi (Napoli, 25-26 novembre 1996), a cura di Maria Luisa Silvestre e Marisa Squillante, Napoli, 1997, p. 301-346.

NAZZARO Antonio V., « La parafrasi agiografica nella tarda Antichità », in Scrivere di santi, a cura di Gennaro Luongo, Roma, 1998, p. 69-107. 
NAZZARo Antonio V., Poesia biblica come espressione teologica : fra tardoantico e altomedioevo, in La Scrittura infinita. Bibbia e poesia in èta medievale e umanistica, Atti del Convegno di Firenze, 26-28 giugno 1997, a cura di F. Stella, Firenze, 2001, p. 119-153.

NAZZARO Antonio V., « Riscritture metriche di testi biblici e agiografici in cerca del genere negato », Auctores nostri,4 (2006), p. 397-439.

NODES Daniel J., Doctrine and Exegesis in Biblical Latin Poetry, Leeds, 1993.

La réécriture hagiographique dans l'Occident médiéval. Transformations formelles et idéologiques, sous la direction de M. Goullet et M. Heinzelmann, Beihefte der Francia, 58, Ostfildern, 2003.

REYDELLET Marc, « Venance Fortunat et l'esthétique du style », in Haut Moyen Âge : culture, éducation et société, Mélanges Pierre Riché, La Garenne-Colombes, 1990.

ROBERTS Michael, Biblical Epic and Rhetorical Paraphrase in Late Antiquity, Liverpool, 1985.

ROBERTS Michael, «The Last Epic of Antiquity : Generic Continuity and Innovation in the Vita Sancti Martini of Venantius Fortunatus ", Transactions of the American Philological Association, 131 (2001), p. 257-285.

ROBERTS Michael, « Venantius Fortunatus's life of saint Martin », Traditio, 57 (2002), p. 129-187.

La réécriture hagiographique dans l'Occident médiéval. Transformations formelles et idéologiques, sous la direction de M. Goullet et M. Heinzelmann, Beihefte der Francia, 58, Ostfildern, 2003.

SPRINGER Carl P.E., The Gospel as Epic in Late Antiquity. The Paschale Carmen of Sedulius, Leyde, 1988.

THRAEDE Klaus, art. “ Epos ”, RLAC, 5, Stuttgart, 1962, col. 997-1043.

WITKE Charles, Numen Litterarum. The Old and the New in Latin Poetry from Constantine to Gregory the Great, Leyde, 1971.

ZARINI Vincent, Rhétorique, poétique, spiritualité: La technique épique de Corippe dans la Johannide, « Recherches sur les rhétoriques religieuses», Turnhout, 2003.

ZARINI Vincent, « Récriture épique et hagiographie martinienne : la Vita Martini de Paulin de Périgueux ", dans Palimpsestes épiques. Récritures et interférences génériques, éd. D. BOUTET et $\mathrm{C}$. ESMEIN-SARRAZIN, Paris, 2006, p. 177-202.

\section{ANNEXES}

\section{Textes}

Paulin de Périgueux, chant 1, v. 317-386 :

(édition et traduction inédites de S. Labarre)

Quidam tam clarae cernens miracula uitae elegit tanto semet sociare magistro, mystica quem nondum fontis renouauerat unda.

320 Huic febris totas penitus depasta medullas extorsit tenuem consumpto corpore uitam. Ac tum forte absens doctor ; sed sedula fratrum religio exanimum plangebat maesta cadauer, maerens supplicio fratris, non funere carnis.

325 Adueniens sanctus fletu rigat ubere uultum, 
defuncti tristem casum miseratus, et omnem

impleuit clamore locum. Mox teste remoto irrupit maestam tam tristi funere cellam.

Tum super exanimum sese proiecit amicum,

330 cunctis frigentes membris complectitur artus aspirans tenuem super ora rigentia flatum.

Attamen ad Dominum penitus spes tota refertur :

assueta oratur pietas, miseratio nota poscitur et meritum fidei credendo meretur.

335 Vidit adesse Deum puri fiducia cordis effectumque piis senserunt uiscera fibris. Nec mora ; uix geminis uotum suspenditur horis, paulatim incussis motantur lintea membris arentesque artus uenarum umore rigantur

340 afflatuque animae pectus glaciale tepescit, obducti infuso reserantur lumine uisus :

felices, quibus indulti sub luminis ortu contigit in primo Martinum cernere mundo. Erigitur totum subito molimine corpus

345 et cutis exesis dudum iam lurida membris purpureum recipit suco meliore ruborem. Vox facilis, gressus stabilis, manus apta, uigens cor : totus homo ex uariis conflato corpore causis unam suscepit diuerso in munere uitam.

350 Eliciunt magnam mox gaudia maxima uocem : laudes lingua sonat gratantis nuntia mentis, affectum cordis clamor pronuntiat oris. Irrupit mox turba fores cellamque repleuit, et sancti cecinit concors symphonia laudes.

355 Ille autem, ut penitus uitam post fata nouaret, confestim aeterna nituit renouatus ab unda et multos felix senium protraxit in annos, tam clari testis non paruo tempore facti. Saepe etenim multis idem narrare solebat

360 iudicis aeterni semet tremuisse tribunal uilibus addictum turbis mersumque tenebris, angelicis donec prolata oratio uerbis offerret uotum Domino uitamque referret. Hic primus nostris Martinum prodidit oris ;

365 hoc aditu ad reliquas peruenit gloria laudes.

Un homme, témoin des miracles d'une vie si illustre, choisit de s'attacher à un si grand maître ; l'onde mystique de la Source ne l'avait pas encore régénéré. (320) Une fièvre qui l'avait entièrement dévoré jusqu'aux moelles extirpa sa faible vie, après avoir consumé son corps. Et à ce moment-là, il se trouvait que le docteur était absent : mais la piété empressée de ses frères pleurait avec affliction sur le cadavre inanimé, s'affligeant du supplice d'un frère, non de la mort de sa chair. (325) À son arrivée, le saint baigne de larmes abondantes son visage, pris de compassion pour le malheur funeste du défunt, et emplit de ses cris tous les lieux. Puis, sans témoin, il fit irruption dans la cellule affligée par une si funeste mort. (330) Alors il se jeta sur son ami privé de vie ; il embrasse de tous ses membres le corps glacé, exhalant un faible souffle au-dessus de sa bouche raidie. Cependant c'est vers le Seigneur que son espoir tout entier se reporte : l'habituelle pitié est sollicitée, la miséricorde bien connue est réclamée, et c'est en croyant que Martin mérite ce que lui mérite sa foi. (335) La confiance de son cœur pur a vu que Dieu était là, et sa chair en a senti l'effet dans ses pieuses entrailles. Et sans retard, la réalisation de son 
vœu n'est suspendue qu'à peine deux heures : peu à peu les membres du défunt se secouent, le linceul s'agite, ses membres desséchés sont baignés par le sang de ses veines, (340) le souffle de la vie commence d'attiédir sa poitrine glacée, ses yeux fermés s'ouvrent, tandis que la lumière s'y répand : heureux furent-ils, eux qui eurent la chance, à la naissance de la lumière qui leur était accordée, de distinguer Martin au commencement du monde. Tout le corps se redresse, dans un effort soudain, et (345) la peau, depuis longtemps déjà livide sur ses membres décharnés, reprend, sous l'effet d'une sève meilleure, un rose pourpré. La parole est aisée, le pas sûr, la main habile, le cœur vigoureux : l'homme tout entier, avec son corps formé de l'assemblage de différents principes, reçut une vie une en ses fonctions diverses. (350) Sa très grande joie fait bientôt entendre un grand cri : sa langue, messagère de son esprit reconnaissant, fait résonner des louanges, le cri de sa bouche proclame les transports de son cœur. Bientôt la foule força les portes et emplit la cellule, et un concert chanta à l'unisson les louanges du saint. (355) Quant à l'homme, afin de mener après l'arrêt du destin une vie tout à fait nouvelle, il se mit soudain à resplendir, renouvelé par l'onde éternelle, et durant de nombreuses années, dans le bonheur, il prolongea sa vieillesse, longtemps témoin d'un exploit aussi illustre. En effet, le même homme avait coutume de raconter souvent à de nombreuses personnes qu'il avait (360) tremblé devant le tribunal du Juge éternel, été voué à de viles multitudes et plongé dans les ténèbres, jusqu'à ce que la prière de Martin, transmise par la voix des anges, présentât son vœu au Seigneur et lui rendît la vie. C'est lui qui, le premier, a révélé Martin à nos contrées ; (365) par cette voie, la gloire du saint parvint jusqu'aux louanges qui s'ensuivirent.

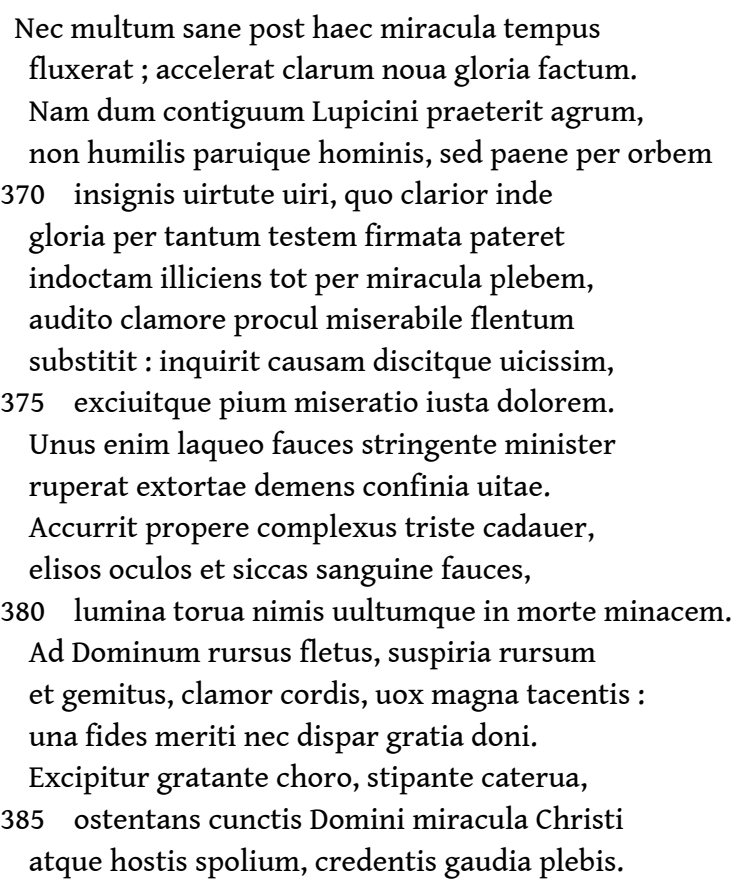

Peu de temps, assurément, s'était écoulé après ce miracle ; la gloire nouvelle de Martin hâte la réalisation d'une illustre action. En effet, celui-ci passe à proximité du domaine de Lupicinus, homme ni humble ni modeste, mais (370) d'une valeur remarquable presque dans le monde entier ; afin qu'à partir de là, avec plus d'éclat, la gloire de Martin, confirmée par un si grand témoin, s'étendît en attirant par de si nombreux miracles une foule ignorante, le saint, après avoir entendu au loin le cri de gens qui pleurent de manière pathétique, s'arrête : il en demande la raison, l'apprend en retour, (375) et sa juste commisération lui tira une pieuse douleur. Un serviteur, en effet, serrant sa gorge 
dans un nœud coulant, avait franchi, dans sa folie, les limites de la vie et se l'était arrachée. Martin accourt en hâte et embrasse le funeste cadavre, les yeux exorbités et la gorge tarie de sang, (380) le regard extrêmement farouche et le visage menaçant dans la mort. Vers le Seigneur de nouveau des pleurs, de nouveau des soupirs et des gémissements, le cri de son cœur, la grande voix de son silence : même confiance dans le mérite, égale grâce dans le don. Il est accueilli par les congratulations d'un chœur, par un cortège qui l'entoure, (385) montrant à tous les miracles du Christ, notre Seigneur, et la dépouille de l'Ennemi, qui font la joie du peuple croyant.

\section{NOTES}

1. V. Zarini (2006) a confronté la Vie de saint Martin de Paulin aux critères définis par W. Kirsch (1989).

2. F. Dupont, Le plaisir et la loi, du banquet de Platon au Satiricon, Paris, 2002, p. 14.

3. Roberts (2001), p. 267.

4. Roberts (2001), p. 258, Roberts (2002), p. 187 (à propos de Fortunat) : «Generically the VSM is an epic $"$.

5. F. E. Consolino (2005) annonce d'emblée: «Aperto è anche il dibattito sullo statuto della parafrasi biblica, che non tutti riconoscono come un genere letterario a tutti gli effetti », p. 449 ; mais elle conclut finalement : «Le parafrasi bibliche invece - anche le più fedeli - non fosse che per l'inevitabile, ulteriore translatio da prosa a poesia, non corrono di questi rischi e possono comunque rivendicare a se stesse uno spazio di autonomia e creatività letteraria », p. 526.

6. A. V. Nazzaro (2006), p. 439: «L'esame dei testi programmatici, sopra effettuato, ha mostrato... che i nostri poeti nella loro attività parafrastica applicavano le norme di Quintiliano $\mathrm{e}$ avevano una piena coscienza letteraria ».

7. S. Labarre, «La composition de la Vita Martini de Sulpice Sévère », Vita Latina 171 (décembre 2004), p. 102-120.

8. Virgile, Énéide, 8, 389-390: «accepit solitam flammam notusque medullas /intrauit calor et labefacta per ossa cucurrit ». Voir aussi Catulle, 35, $15:$ « ignes interiorem edunt medullam »; 45, 16 : « ignis mollibus ardet in medullis »;64, $93:$ « funditus atque imis exarsit tota medullis ».

9. Lucrèce, 6, 1224 : "extorquebat enim uitam uis morbida membris » (peste d'Athènes); Ambroise, Hexameron, 6, 8, 48, CSEL, 32, 1, p. 239, 3 : « qui uitam quam deus donauit extorquent »; Augustin, Lettres, 134, 4, CSEL, 44, p. 88, 5 : « extorserunt spatium uiuendi »; Orose, Histoires, 1, 20, $6:$ : innocentum uitas poenis torquentium ».

10. Paulin de Périgueux, Vie de saint Martin, 1, 377 : " ruperat extortae demens confinia uitae ».

11. Paulin de Périgueux, Vie de saint Martin, 1, 322-324: « sed sedula fratrum religio exanimum plangebat maesta / cadauer, maerens supplicio fratris, non funere carnis. »

12. Paulin de Périgueux, Vie de saint Martin, 1, 325 : «Fletu rigat ubere uultum »; Virgile, Énéide, 6,699 : « largo fletu simul ora rigabat ».

13. Paulin de Périgueux, Vie de saint Martin, 1, 326-327 : « ... et omnem impleuit clamore locum »; Virgile, Énéide, 3, 313-314.

14. Paulin, Vie de saint Martin, 1, 378 sv.: «Accurrit propere complexus triste cadauer / elisos oculos et siccas sanguine fauces »; Virgile, Énéide, 8, 259-261: «Hic Cacum in tenebris incendia uana uomentem / corripit in nodum complexus et angit inhaerens / elisos oculos et siccum sanguine guttur ».

15. Prudence, Psychomachie, 32.

16. Sulpice Sévère, Vie de saint Martin, 7, 3: « sanctum spiritum concipiens »; Lucain, 5, 163 : « haesit et inuito concepit pectore numen ». 
17. Paulin, Vie de saint Martin, 1, 251-252 et Virgile, Énéide, 9, 391 sv.

18. Arator, De actibus apostolorum, 1,$37 ; 2,1117$ : «rector Olympi »; 1, 49, 613, $824 ; 2,145$ : «Tonans »;1, 183, $623 ; 2,481,796,815$ : « Tartarus »; 1, 37, 111, $346 ; 2,289,1117$ : « Olympus ».

19. Thraede (1962) col. 1025 ; Green (2006) p. 23, 95, 171, 204, 207.

20. Prudence, Apotheosis, 756 : « euomit exequias gradiente cadauere uiuas ».

21. Fortunat, Vie de saint Martin, 1, 187-188: «mors pede uersa retro praedam fugitiua reliquit / euomuitque uorax auida de fauce rapinam ».

22. Charlet, 2008, p. 161.

23. Cutino (2009), p. 185-206.

24. Sulpice Sévère, Vie de saint Martin, 7, 3: «cum...sensisset per spiritum Domini adesse uirtutem ».

25. Sulpice Sévère, Vie de saint Martin, 7, 3: «orationis suae ac misericordiae Domini intrepidus expectabat euentum ».

26. Luc, 5, 17 : « et uirtus Domini erat »; Actes, 1, 8 : « uirtutem...Spiritus Sancti ».

27. 1 Rois 17, 21 et 2 Rois 4,34 .

28. 1Rois, 17, 21 : « et expandit se atque mensus est super puerum tribus uicibus ».

29. 2 Rois, 4, 33-34: "(Heliseus) ingressusque clusit ostium super se et puerum et orauit ad Dominum et ascendit et incubuit super puerum posuitque os suum super os eius et oculos suos super oculos eius et manus suas super manus eius et incuruauit se super eum et calefacta est caro pueri».

30. Ambroise, Lettres, 4, 17 (81), 5, CSEL 82, 1, p. 124 : « Unde in Helisaeo figura Domini praecessit, qui proiecit se, ut mortuum erigeret. In quo symbolum est quod nobis Christus commortuus sit, ut nobis resurgeret "; Jérôme, Comment. in Eccl., IV, 9/12, CCL 72, p. 288, 1. 157-162 : « De Elisaeo sumamus exemplum, quod et ipse contraxerit se cum puero et dormierit et calefecerit corpusculum eius et ita uiuificauerit resurgentem. Nisi igitur Christus nobiscum dormierit et in morte requieuerit calorem aeternae uitae accipere non ualemus ».

31. Matthieu, 9, 18.

32. Luc, $7,11$.

33. Actes, 9,36 .

34. Vulgate, Jean, 3, 2: " a Deo uenisti magister »; Tertullien, Scorpiace, CC 2, 9, 6 ; Cyprien, De bono patientiae, CC 3 A, 13 ; Augustin, Cité de Dieu, BA 37, 21, 27, 548 ; Prudence, Apotheosis, 707.

35. Tertullien, Les spectacles, SC 332, 21, 1 : « doctor ueritatis »; Ps. Hilaire, Carmen de Euangelio, 101 : «doctor uerus »; Lactance, Institutions divines, SC, 326, 1, 1, 19: «doctorem sapientiae » ;Juvencus, CSEL 24, 2, 352 ;Prudence, Psychomachie, 888 ;Sédulius, Carmen paschale, CSEL 10, 3, 322 et Opus paschale, CSEL 10, 2, 14, p. 218, 10.

36. Paulin, Vie de saint Martin, 1, 341-343 : «obducti infuso reserantur lumine uisus :/ felices, quibus indulti sub luminis ortu / contigit in primo Martinum cernere mundo ».

37. Paulin, Vie de saint Martin, 1, $340:$ « afflatuque animae pectus glaciale tepescit ».

38. Tertullien, Contre Marcion, SC 368, 2, 9, 1: «afflatus Dei, id est anima »; Genèse, 2, 7 : « et inspirauit (Deus) in faciem eius spiraculum uitae ».

39. Art. « Oculus » et art. « Sens spirituels », Dictionnaire de spiritualité, Paris, 1932-1995.

40. Origène, Contre Celse, 7, 33, SC 150, p. 88.

41. Paulin de Périgueux, Vie de saint Martin, 1, 347 : «uox facilis, gressus stabilis, manus apta, uigens cor" $"$.

42. Paulin de Périgueux, Vie de saint Martin, 1, 348-349: «totus homo ex uariis conflato corpore causis / unam suscepit diuerso in munere uitam ».

43. Augustin, Confessions, 10, 7, 11 : « quae diuersa per eos ago unus ego animus ».

44. Reydellet (1990), p. 76. 
45. Prudence, Cathemerinon, 3, 204-205: «dux parili rediuiuus humo / ignea Christus ad astra uocat "; Cyprien, La bienfaisance et les aumônes, SC 440, 6: "rediuiuum corpus animatur»; Sédulius, Opus paschale, 4, 21, CSEL 10, p. $271:$ « rediuiua animatione ».

46. Ambroise, Expositio psalmi 118, 10, 6, CSEL 62, p. 206: «fabricam ipsam humani corporis si quis considerare uelit, nihil poterit in terra pretiosius iudicare ";Prudence, Livre d'heures, 11, 43-44: « inpune ne forsan sui / Patris periret fabrica »;Fortunat, Vita sancti Germani, 45, 126, MGH, AA, IV, 2 , p. 21 : «naturalibus motibus ad uitalem usum tota fabrica renouatur ».

47. Fortunat, Vie de saint Martin, 1, 198-199: « usque ad uestibulum domus illum ducere tendens,/ defunctum eripuit subito qui ex limine mortis ».

48. Fortunat, Vie de saint Martin, 1, 176: « ipse iterum post se uiuens, idem auctor et heres ».

49. Fortunat, Vie de saint Martin, 1, 105-107 : « illa istum mundo, hic illam generauit Olympo /...et meliore sinu generant sua uiscera matrem ».

50. Paulin de Périgueux, Vie de saint Martin, 1, 233-234: «...unoque et mater et infans / facta puerperio ! genetrix generanda ».

51. Sédulius, Carmen paschale, 2, 40, CSEL 10, p. 47 : «Virgo sinus gaudetque suum paritura parentem »

\section{RÉSUMÉS}

Le récit hagiographique se prête particulièrement bien à la réécriture épique. Paulin de Périgueux remploie maintes réminiscences de Virgile, parfois de Lucrèce ou de Lucain. Les épopées latines du Nouveau Testament ont aussi eu une grande influence sur les poètes hagiographiques. En fait, les épopées hagiographiques et bibliques constituent un sous-genre à l'intérieur du genre épique. Paulin insiste sur l'enseignement moral de chaque épisode, tandis que Fortunat pratique une écriture épigrammatique, même dans son poème hagiographique. Ils s'inscrivent dans la tradition de la méditation poétique, mais d'une manière différente. L'épopée hagiographique a des ambitions littéraires. Son but est de charmer, émouvoir et instruire, et si possible de rivaliser avec l'épopée antique.

The life of a saint is evidently an appropriate material for epic treatment. Paulinus' poem incorporates many reminiscences of Vergil, sometimes of Lucretius and Lucan. Latin epics of the New Testament also had a great influence on the hagiographical poets. In fact hagiographical and biblical epics constitute a subgenre, a special variety of epic. Paulinus emphasizes the teaching of an episode, while the epigrammatic quality of Fortunatus' poetry is quite evident, even in his hagiographical poem. They both develop the tradition of poetic meditation, but in their different ways. Hagiographical epic has literary ambitions. Its purpose is to delight, to move and to instruct, and if possible to rivalize with the ancient epic.

\section{INDEX}

Mots-clés : Bible, épopée, hagiographie, Paulin de Périgueux, Venance Fortunat, Virgile 


\section{AUTEUR}

\section{SYLVIE LABARRE}

Maître de conférences en langue et littérature latinesà l'Université du Maine (Le Mans), membre statutaire de l'Institut d'Études Augustiniennes(Laboratoire d'Études sur les Monothéismes CNRS UMR 8584), associé au Labo 3LAM (Université du Maine).

Agrégation de Lettres classiques (1987).

Doctorat en littérature latine à l'Université Paris-Sorbonne, dir. Jean-Claude Fredouille, (1994). 